\title{
Efeito da razão de refino de malha sobre o uso de multiextrapolação de Richardson em CFD
}

\author{
$\underline{\text { Fabiana de Fátima Giacomini }}^{\text {¿ }}$, Carlos Henrique Marchi ${ }^{\#}$, \\ Universidade Federal do Paraná (UFPR) - Programa de Pós-Graduação em Engenharia Mecânica ${ }^{\&}$ \\ Universidade Federal do Paraná (UFPR) - Departamento de Engenharia Mecânica\# \\ 81531-980, Curitiba, PR \\ E-mail: giacomini@ufpr.br ${ }^{\star}$, marchi@ufpr.br ${ }^{\#}$
}

\begin{abstract}
Resumo: O objetivo deste trabalho é estudar o efeito da razão de refino de malha sobre multiextrapolações de Richardson (MER) em problemas uni e bidimensionais resolvidos com diferenças finitas e volumes finitos. Foram empregados três modelos numéricos: Poisson 1D, Laplace e Burgers 2D, discretizados com aproximação numérica de $2^{a}$ ordem. Foram utilizados conjuntos de malhas base com 2, 4, 64, 256 e 1024 elementos iniciais. Empregou-se razões de refino iguais a 1,2, $1,25,1,5,2,3,4$ e 5. Foi utilizado variação constante (DN) entre os elementos da malha, sendo iguais a 2, 4, 8, 16 e 32 elementos. Também foram empregadas razões de refino variáveis (Dr) iguais a 0,5 e 1. Verificou-se que: independente da escolha da razão de refino e da malha base o erro numérico diminui após aplicar MER; usar DN constante entre as malhas pode ser uma alternativa na geração de conjuntos de malhas; a razão de refino igual a 2 apresentou o menor erro após aplicar MER; $e$ malha base com maior número de elementos apresenta maior ordem efetiva.
\end{abstract}

Palavras-chave: erro de discretização, ordem efetiva, multiextrapolação de Richardson, razão de refino, malha base.

\section{INTRODUÇÃO}

Do ponto de vista matemático, uma solução numérica ideal deve ter erro numérico nulo, ou seja, deve resultar na solução analítica. Portanto, estudar técnicas que sejam eficientes na redução do erro numérico ou de suas fontes é importante para que seja possível medir adequadamente o erro de modelagem, isto é, o erro de um modelo matemático usado para representar um fenômeno físico real.

Por isso, para reduzir o erro numérico causado por erros de discretização foi estudado o efeito da razão de refino de malha, que segundo [5] é um parâmetro que afeta diretamente a estimativa do erro de discretização feita pelos estimadores mais empregados na literatura. Devido ao emprego do estimador GCI (Grid Convergence Index), o qual relata o grau de refinamento da malha, foi constatado por [1] que estudos futuros são necessários para avaliar analiticamente a influência da geometria das células na acurácia da solução e mais precisamente avaliar outras opções de sistemas de malhas incluindo várias formas de configuração de malhas híbridas.

Assim, para estudar o efeito da razão de refino na redução do erro de discretização em várias malhas com $h$ diferentes empregou-se MER (Multiextrapolação de Richardson), que é um estimador baseado na extrapolação de Richardson [3] e que foi desenvolvido por [2] para reduzir e estimar o erro de discretização da equação de Laplace 2D empregando somente razão de refino igual a 2.

\section{MODELOS MATEMÁTICOS}

O modelo matemático unidimensional (1D) é a equação de Poisson [4], expressa por

$$
\frac{d^{2} u}{d x^{2}}=-\pi^{2} \operatorname{sen}(\pi x)
$$

onde $u$ representa a variável dependente e $x$ é a coordenada espacial. As condições de contorno, do tipo Dirichlet, são $u(0)=u(1)=0$. Sua solução analítica é $u=\operatorname{sen}(\pi x)$.

O primeiro modelo matemático bidimensional (2D) é a equação de Laplace, expressa por 
$\frac{\partial^{2} u}{\partial x^{2}}+\frac{\partial^{2} u}{\partial y^{2}}=0$

onde $y$ é a $2^{\mathrm{a}}$ coordenada espacial. As condições de contorno e as soluções analíticas são dadas em [2].

As equações de Burgers são o segundo modelo matemático 2D. Elas podem ser expressas por

$u \frac{\partial u}{\partial x}+v \frac{\partial u}{\partial y}=-\frac{\partial p}{\partial x}+\frac{1}{\operatorname{Re}}\left(\frac{\partial^{2} u}{\partial x^{2}}+\frac{\partial^{2} u}{\partial y^{2}}\right) \quad$ e $\quad u \frac{\partial v}{\partial x}+v \frac{\partial v}{\partial y}=-\frac{\partial p}{\partial y}+\frac{1}{\operatorname{Re}}\left(\frac{\partial^{2} v}{\partial x^{2}}+\frac{\partial^{2} v}{\partial y^{2}}\right)-S(x, y, \operatorname{Re})$

onde $v$ representa a segunda variável dependente; $p$ é a pressão, conhecida analiticamente; Re é o número de Reynolds; e $S$ é um termo fonte. As variáveis $p$ e $S$, as condições de contorno e as soluções analíticas são dadas em [6].

\section{MODELOS NUMÉRICOS}

A solução numérica sem extrapolação foi obtida pelo método de diferenças finitas [7], para Poisson e Laplace, e de volumes finitos [8], para Burgers. A discretização foi realizada em malhas estruturadas e uniformes. Para os três modelos matemáticos foi considerada a aproximação CDS-2 (Central Differencing Scheme) de $2^{\mathrm{a}}$ ordem. O solver utilizado para Poisson foi o TDMA (TriDiagonal Matrix Algorithm). Para Laplace foi o MSI (Modified Strongly Implicit) e para Burgers foi o Gauss-Seidel, ambos com o acelerador de convergência multigrid [9]. Os programas foram implementados em linguagem FORTRAN 95, versão Intel 11.1.065, e precisão quádrupla.

A solução numérica $(u)$ em cada malha $g$ com $m$ extrapolações de Richardson, para cada variável de interesse, é dada por

$u_{g, m}=u_{g, m-1}+\frac{u_{g, m-1}-u_{g-1, m-1}}{r^{p_{m-1}}-1}$

onde $p_{m}$ representa as ordens verdadeiras [2] do erro de discretização; $h$ é a distância entre dois nós consecutivos; $r=h_{g-1} / h_{g}$ é a razão de refino; $g=[2, G]$, onde $g=1$ é a malha mais grossa, e $g=G$ é a malha mais fina; e $m=[1, \mathrm{~g}-1]$ é o número de extrapolações, onde $m=0$ significa que a solução não apresenta qualquer extrapolação.

\section{RESULTADOS}

O erro de discretização da solução numérica em cada nó $i$ é definido por

$$
E_{i}=u_{i}^{\text {analitica }}-u_{i}^{\text {numérica }}
$$

Para analisar a eficiência de MER na redução do erro de discretização de um conjunto de malhas, foi empregado o conceito de ordem efetiva, calculada por [2]

$\left(p_{E}\right)_{g, m}=\frac{\log \left(\frac{E_{g-1, m-1}}{E_{g, m}}\right)}{\log (r)}$

onde $g=[2, G]$ e $m=[1, g-1]$.

A equação de Laplace apresenta resultados para a variável de interesse $u$ em $x=1 / 2$ e $y=1 / 2\left(u_{c}\right)$, ou seja, no centro do domínio de cálculo. Foram simuladas para essa variável cinco conjuntos de 
malhas com diferentes razões de refino: para $r=2$, foram obtidas 11 malhas com 3, 5, 9, 17, 33, 65, $129,257,513,1025$ e 2049 nós por direção; para $r=4$, foram obtidas 7 malhas com 3, 9, 33, 129, 513, 2049 e 8193 nós por direção; para $r=3$, foram obtidas 7 malhas com 3, 7, 19, 55, 163, 487 e 1459 nós por direção; para $r \approx 1,5$ foram obtidas 14 malhas com 3, 5, 7, 11, 17, 25, 37, 55, 83, 123, 183, 273, 409 e 613 nós por direção; e para $r \approx 1,2$ foram obtidas 22 malhas com 3, 5, 7, 9, 11, 13, 15, 19, 23, 27, 31, 37, 43, 51, 61, 73, 87, 103, 123, 147, 175 e 209 nós por direção.

Pela Figura 1 percebe-se que os resultados extrapolados (Em) apresentam os menores erros com relação aos resultados sem extrapolação (Eh), usando qualquer uma das cinco razões de refino. $\mathrm{O}$ menor erro é obtido com $r \approx 1,2$ até $h=3,125 \times 10^{-2}$. Abaixo de $h=3,125 \times 10^{-2}$, a redução com $r=2$ é bem menor do que as demais razões de refino.

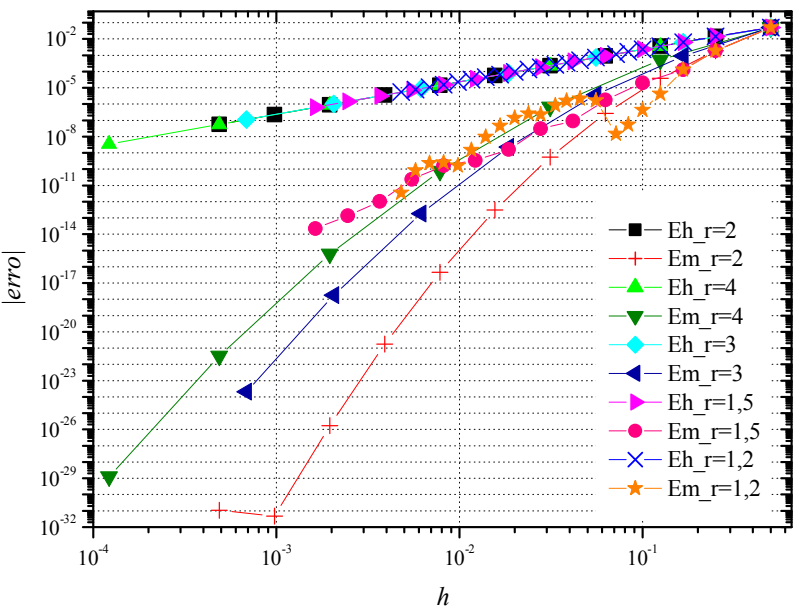

Figura 1: $u_{c}$ - equação de Laplace 2D

Para a equação de Poisson foram obtidos resultados para $u$ no centro do domínio de cálculo $\left(u_{c}\right)$, a média de $u$ e fluxo de calor em $x=1$. Para Burgers foram simuladas as variáveis: velocidade no centro do domínio de cálculo $\left(u_{c}\right)$, fluxo de massa, força de arrasto na parede inferior, valor mínimo da componente da velocidade na direção horizontal e valor mínimo da função corrente do domínio inteiro. O presente artigo mostra resultados somente para a variável de interesse $u_{c}$ devido ao volume de dados e resultados das demais variáveis.

A Figura 2 apresenta o módulo do erro numérico para a equação de Poisson. Foram simuladas quatro razões de refino em três conjuntos de elementos iniciais diferentes. Ou seja, quando a malha mais grossa iniciava com $\mathrm{N}=2$ elementos, foram empregados quatro conjuntos de malhas com razões de refino iguais a 2, 3, 4 e 5. Da mesma forma, quando a malha mais grossa iniciava com $\mathrm{N}=64$ elementos, e também, quando considerava-se a malha mais grossa iniciando com $\mathrm{N}=1024$ elementos. Percebe-se que as soluções extrapoladas (Em) apresentam menores erros com relação às soluções sem extrapolação (Eh) indiferente da escolha da razão de refino. As curvas Eh_N2_r=2 e Eh_N1024_r=5 representam as soluções sem extrapolação, visto que as demais curvas sem extrapolação apresentam resultados qualitativamente iguais. Em $h=9,76 \times 10^{-4}$, a curva Em_N2_r=2 apresentou o menor erro numérico $\left(7,40 \times 10^{-32}\right)$ com 9 extrapolações. Em $h=2,28 \times 10^{-4}$, a curva Em_N2_r=3 apresentou um erro igual a $3,71 \times 10^{-32}$ e 7 extrapolações. A partir de certo $h$, os erros das extrapolações começam a aumentar. Esse aumento se dá pelo erro de arredondamento de máquina.

A Figura 3 apresenta a ordem efetiva do erro numérico para a equação de Poisson 1D. A ordem assintótica (a priori) das curvas sem extrapolação tende a 2. A ordem efetiva da curva Em_N2_r=2 tende a 18 com 7 extrapolações. A ordem efetiva da curva Em_N64_r=2 tende a 22 com 4 extrapolações. E a ordem efetiva da curva Em_N1024_r $=2$ tende a 28 com 3 extrapolações. De forma análoga ocorre com as demais razões de refino. Quanto mais fina inicia-se a malha base, a ordem tende a aumentar seu valor após a malha ser extrapolada. 
Para Poisson também foram simuladas malhas com razões de refino iguais a 1,$25 ; 1,5$ e 2,5 usando malha base com 64 e 1024 nós. O comportamento dos resultados do erro mostraram-se qualitativamente iguais aos resultados da Figura 2. As ordens efetivas apresentadas, usando a malha base com 64 nós, tenderam a: 46 para $r=1,25$ com 2 extrapolações; 34 para $r=1,5$ com 5 extrapolações; e 18 para $r=2,5$ com 4 extrapolações. As ordens efetivas apresentadas, usando a malha base com 1024 nós, tenderam a: 73 para $r=1,25$ com 3 extrapolações; 43 para $r=1,5$ com 3 extrapolações; e 20 para $r=2,5$ com 2 extrapolações.

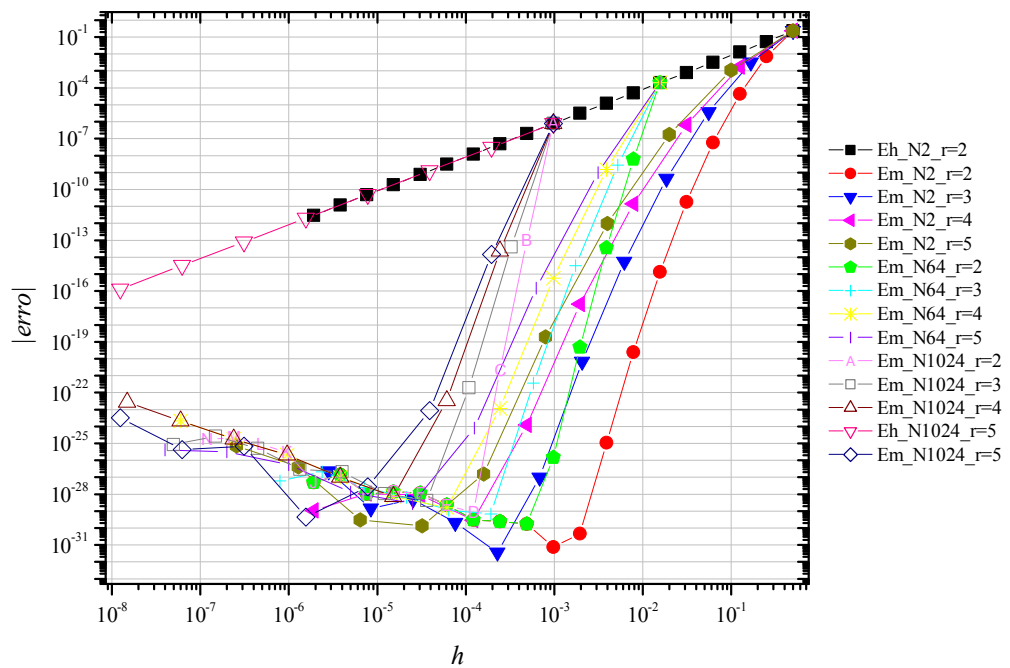

Figura 2: $u_{c}$ - equação de Poisson 1D

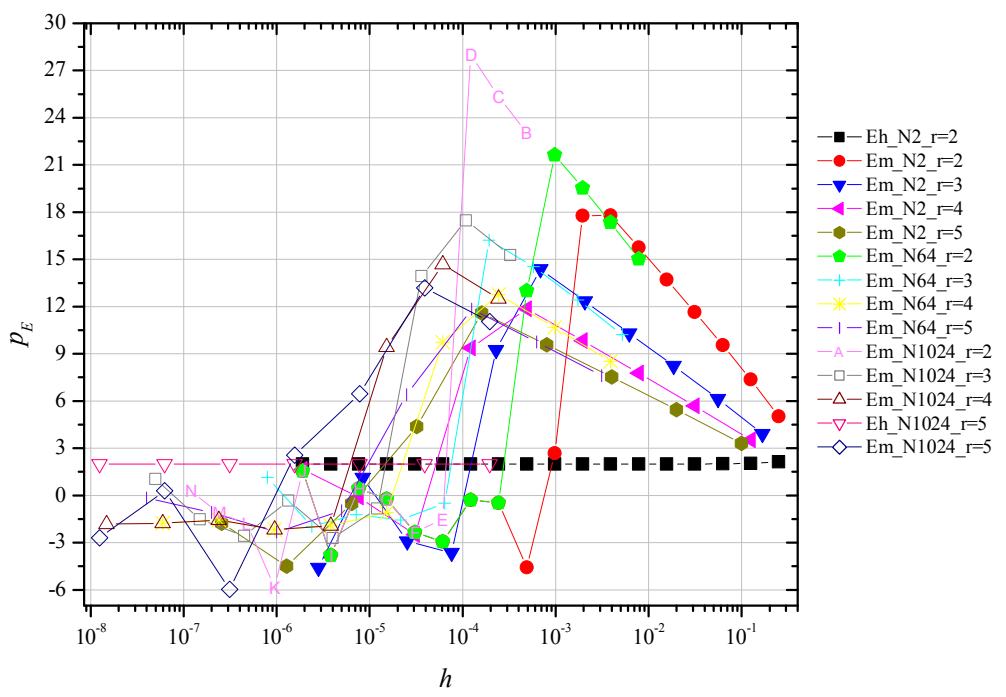

Figura 3: $p_{E}$ - equação de Poisson 1D

Ainda para Poisson, foram simuladas para cada malha base com $\mathrm{N}=2,64$ e 1024 elementos, conjuntos de malhas que apresentam variações nesses elementos entre cada malha subsequente. Por exemplo, se a malha base inicia-se com $\mathrm{N}=2$, os próximos elementos da malha com a variação desses elementos igual a $2(\mathrm{DN}=2)$ são: $4,6,8, \ldots$. Assim, se a malha base começar com $\mathrm{N}=64$, os próximos elementos da malha com $\mathrm{DN}=16$ serão: $80,96,112, \ldots$ Também foram obtidas malhas que apresentam a variação $\mathrm{Dr}=1, \operatorname{com} r$ inicial igual a 2 e $r$ final igual a $8, \mathrm{e} \mathrm{Dr}=0,5, \operatorname{com} r$ inicial $1,5 \mathrm{e} r$ final 5,5. As Figuras 4 e 5 trazem os resultados para a malha base iniciando com 64 elementos. As malhas bases iniciando com 2 e 1024 elementos apresentam resultados qualitativamente iguais aos apresentados a seguir. 
A Figura 4 mostra o módulo do erro das soluções sem extrapolação (Eh) e das soluções multiextrapoladas $(\mathrm{Em})$ para $\mathrm{DN}=2,4,8,16$ e 32, e $\mathrm{Dr}=1$ e 0,5 . As curvas Eh_r=2 e Eh_Dr=0,5 representam as curvas sem extrapolação. As demais curvas Eh apresentam resultados qualitativamente iguais. Percebe-se que todas as soluções extrapoladas apresentam erro numérico menor em relação às soluções sem extrapolação. Entre $h=1,56 \times 10^{-2}$ e $h=2,05 \times 10^{-3}$, a curva Em_r=1,5, que é a curva da solução extrapolada com razão de refino igual a 1,5 , apresenta erro igual a $5,99 \times 10^{-30}$ com 5 extrapolações; enquanto sua solução sem extrapolação tem erro igual a $3,48 \times 10^{-6}$.

A Figura 5 traz as ordens efetivas referentes aos erros apresentados na Figura 4 . A ordem efetiva da curva Em_r=1,25 tende a 46 com 2 extrapolações. A curva $E m \_r=1,5$ tem ordem efetiva tendendo a 34 com 5 extrapolações. Já a curva Em DN2 apresenta ordem tendendo a 295 na primeira extrapolação e 142 na segunda. Nas demais extrapolações a ordem tende a 11.

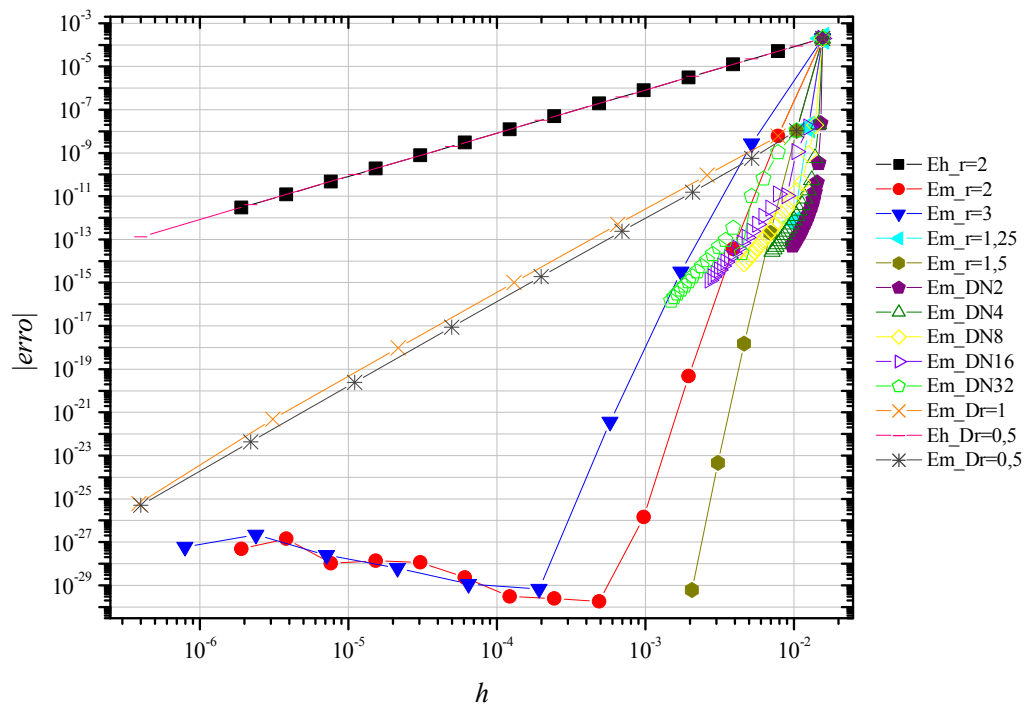

Figura 4: $u_{c}$ - N64 - equação de Poisson 1D

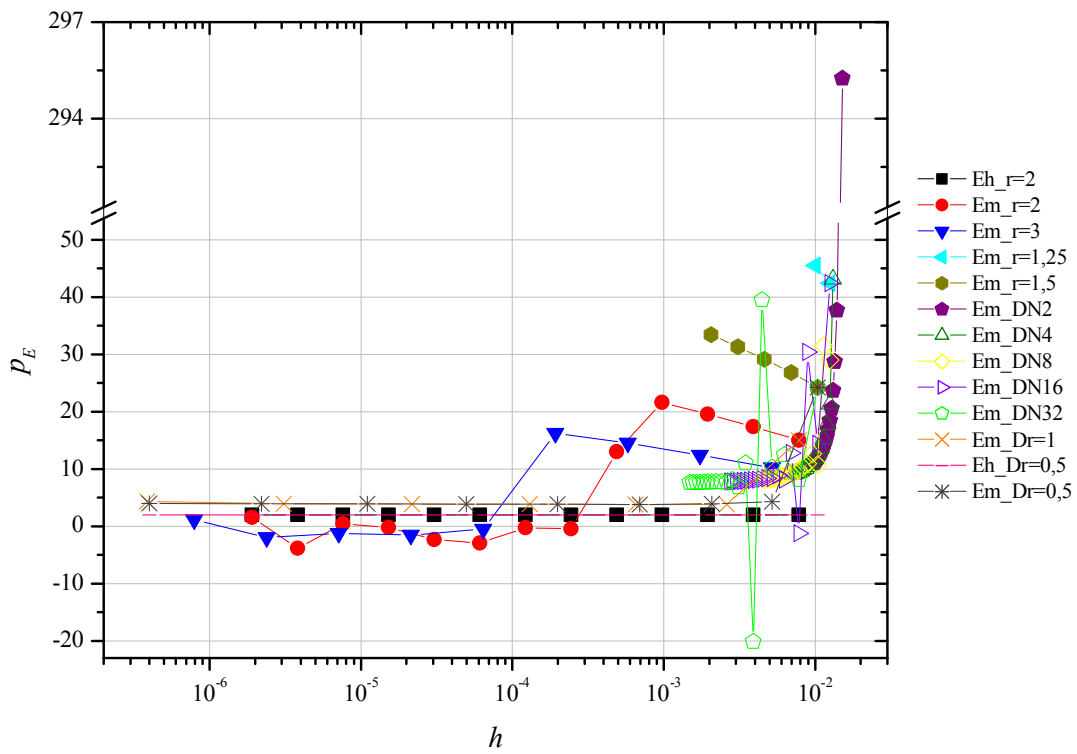

Figura 5: $p_{E}$ - N64 - equação de Poisson 1D

Para a equação de Burgers 2D foram simulados três conjuntos de malhas iniciando com 4, 64 e 256 elementos. Para $\mathrm{N}=4$ empregou-se $r=2$ e $r=3$. Para $\mathrm{N}=64$ e $\mathrm{N}=256$ utilizou-se as razões de refino $r=1,25, r=1,5, r=2 \mathrm{e} r=3$. Os resultados encontram-se nas Figuras 6 e 7 . 
Percebe-se pela Figura 6 que as soluções extrapoladas de qualquer razão de refino apresentam menores erros com relação às soluções sem extrapolação. As curvas Eh_N4_r=2 e Eh_N256_r=3 representam as soluções sem extrapolação, visto que as demais curvas Eh apresentam resultados qualitativamente iguais. Independente de qual malha base comece o conjunto de malhas, a razão de refino igual a 2 apresentou o menor erro numérico nas soluções extrapoladas. No entanto, percebe-se que se fossem adicionados mais níveis de malhas para as razões de refino 1,25 e 1,5 se teriam erros menores nas soluções extrapoladas.

Pela Figura 7, percebe-se que a ordem efetiva a priori das soluções sem extrapolação tende a 2. Os erros que apresentaram as maiores ordens foram os erros (Em) das razões de refino 1,25 e 1,5 com malhas base 64 e 256. Isso pode ser visto pelas curvas: Em_N64_r=1,25 que apresentou ordem tendendo a 22 com 1 extrapolação; Em_N64_r=1,5 com ordem efetiva tendendo a 18 na quarta extrapolação; Em_N256_r=1,25 que apresentou a maior ordem de todas, tendendo a 28 na primeira extrapolação; e Em_N256_r=1,5 com ordem efetiva tendendo a 16 com 3 extrapolações. A curva Em_N64_r=2 teve a ordem efetiva tendendo a 17 com 7 extrapolações.

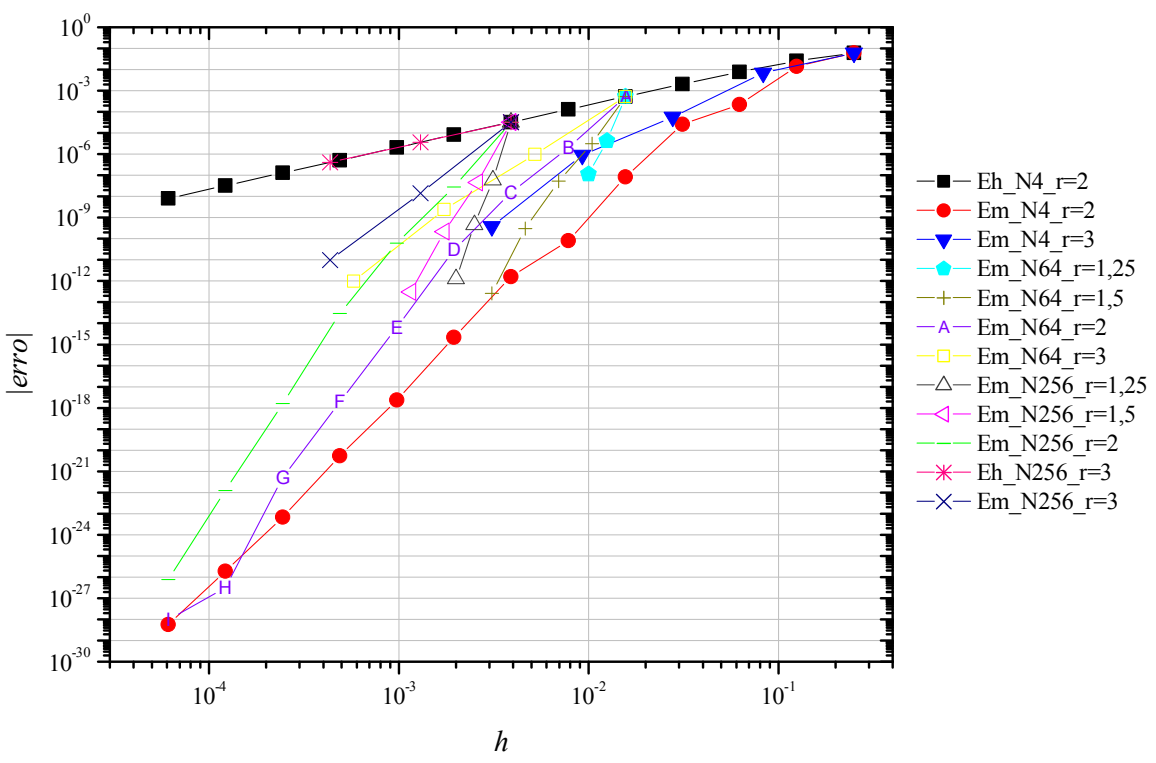

Figura 6: $u_{c}$ - equação de Burgers 2D

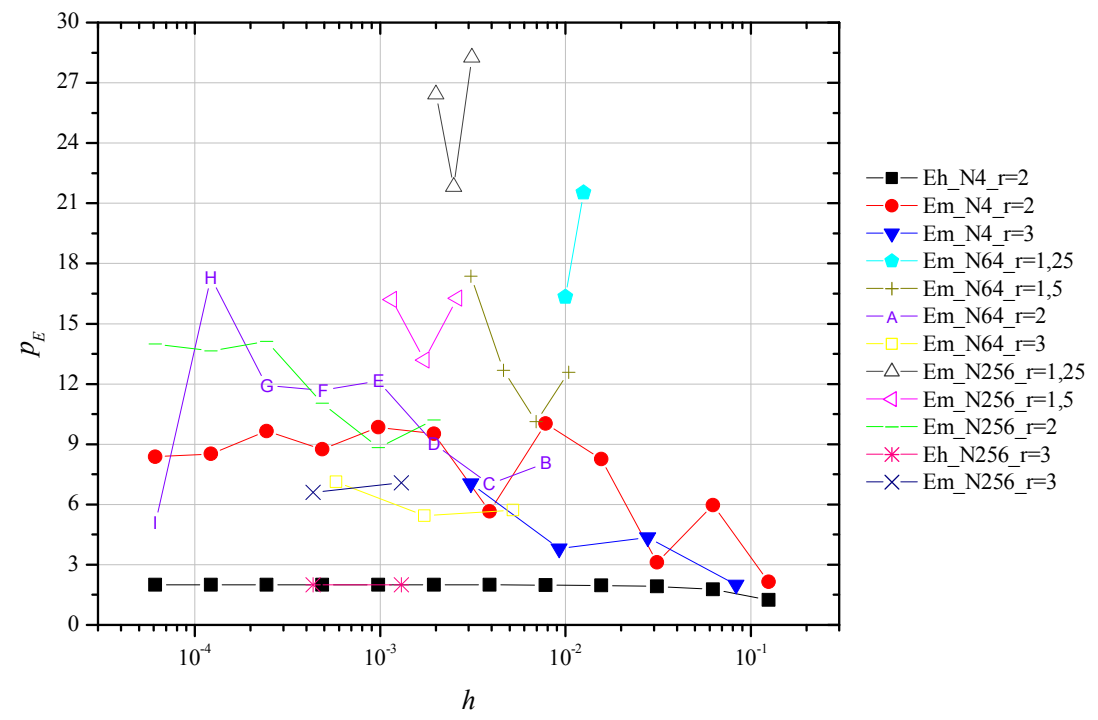

Figura 7: $p_{E}$ - equação de Burgers 2D 


\section{CONCLUSÃO}

Neste artigo foram empregados três modelos numéricos: Poisson 1D, Laplace e Burgers 2D, discretizados com aproximação numérica de $2^{\mathrm{a}}$ ordem em diferenças finitas e volumes finitos. Foram utilizados conjuntos de malhas base com 2, 4, 64, 256 e 1024 elementos iniciais. Empregou-se razões de refino iguais a $1,2,1,25,1,5,2,3,4$ e 5 . Também foi utilizada a variação constante entre os elementos da malha, sendo DN iguais a 2, 4, 8, 16 e 32 elementos.

Constatou-se que: a) independente da escolha da razão de refino, o erro numérico diminui após aplicar MER; b) independente da escolha da malha base, o erro diminui após aplicar MER; c) usar a variação DN constante entre as malhas pode ser uma alternativa na geração de conjuntos de malhas, pois o erro diminui com a aplicação de MER; d) a razão de refino igual a 2 apresentou o menor erro numérico com malhas base iguais a 2, 64 e 1024 elementos; e e) a malha base com maior número de elementos apresenta maior ordem efetiva nas extrapolações iniciais.

\section{AGRADECIMENTOS}

Os autores agradecem o apoio financeiro do CNPq (Conselho Nacional de Desenvolvimento Científico e Tecnológico), AEB (Agência Espacial Brasileira, pelo Programa Uniespaço), CAPES (Coordenação de Aperfeiçoamento de Pessoal de Nível Superior). Agradecem pela implementação do código multigrid aos componentes do grupo de pesquisa CFD, propulsão e aerodinâmica de foguetes da UFPR Cosmo Damião Santiago, Simone de Fátima Tomazzoni Gonçalves e Márcio André Martins. O primeiro autor recebeu bolsa da CAPES. O segundo autor é bolsista do CNPq.

\section{REFERÊNCIAS}

[1] M. M. Hefny, R. Ooka, Influence of Cell Geometry and Mesh Resolution on Large Eddy Simulation Prediction of Flow around a Single Building, Build Simul., 1 (2008) 251-260.

[2] C. H. Marchi, L. A. Novak, C. D. Santiago, A. P. S. Vargas. Highly accurate numerical solutions with repeated Richardson extrapolation for 2D Laplace equation, Applied Mathematical Modelling, 37 (2013) 7386-7397. DOI: 10.1016/j.apm.2013.02.043.

[3] L. F. Richardson, The Approximate Arithmetical Solution by Finite Differences of Physical Problems Involving Differential Equations, with an Application to the Stresses in a Masonry Dam, Phylosophical Proceedings of the Royal Society of London Serial A, 210 (1910) 307-357.

[4] P. J. Roache, P. M. Knupp, Completed Richardson Extrapolation, Communications in Numerical Methods in Engineering, 9 (1993) 365-374.

[5] F. A. Schneider; C. H. Marchi, On the Refinement Ratio for One-Dimensional Advective Problems with Nonuniform Grids, em " $18^{\text {th }}$ International Congress of Mechanical Engineering (XVIII COBEM)", Ouro Preto, 2005.

[6] T. M. Shih, C. H. Tan, B. C. Hwang, Effects of Grid Staggering on Numerical Schemes, International Journal for Numerical Methods in Fluids, 9 (1989) 193-212.

[7] J. C. Tannehill, D. A. Anderson, R. H. Pletcher, "Computational Fluid Mechanics and Heat Transfer", 2 ed, Washington: Taylor \& Francis, 1997.

[8] H. K. Versteeg, W. Malalasekera, "An Introduction to Computational Fluid Dynamics: The Finite Volume Method”, 2 ed, Harlow, England: Pearson/Prentice Hall, 2007.

[9] P. Wesseling, “An introduction to multigrid methods", Wiley, New York, 1992. 